\title{
Editorial
}

\section{All-Electronic Rapid Communications}

With this issue the Journal of Neuroscience launches a pioneering initiative in journal publishing: all-electronc Rapid Communications (RCs). The aim is to publish short articles of timely importance and broad interest as rapidly as possible, using the advantages of electronic publishing.

The first advantage will be a significant reduction in the delay between the time that a manuscript is approved by the editors to the time that it is published in the online journal. The aim is to reduce that time to less than 1 month while still maintaining the high standards of copy editing and data presentation that are associated with the journal. This represents a considerable improvement over the time in press for the hard-copy version, 2-3 months, as in other journals.

The format for the RC articles will be the same as for regular articles. The articles will have a high profile in the journal because they will appear at the top of the table of contents at the beginning of each issue. Citations will indicate these articles by RC. Details regarding submission of articles will be found in the revised Instructions for Authors.

The next improvement will come with all-electronic submission and review of articles. We have been actively investigating this direction during the past year, but the methods are only now being developed that will ensure that these processes are error-free and cost-efficient. We anticipate that this development will reach fruition during the coming year. The benefits will reduce the delay of papers under review even further. In addition, the benefits will accrue to submission of regular papers to the journal.

Although the electronic age has promised developments such as all-electronic publishing for several years, the challenge of working out the details has been enormous to ensure the level of excellence expected by our authors and readers (to say nothing of avoiding the multitude of unexpected annoyances when formats do not work and software is incompatible). The journal welcomes suggestions on solving these problems and moving effectively into the new electronic medium while retaining the advantages and qualities of hard-copy printing.

The basic premise underlying the all-electronic Rapid Communications has been that they meet the same high standards of scholarship that apply to the regular articles in the journal. We will maintain that principle above all else as we take the next steps into the electronic era of journal publishing.

Gordon M. Shepherd Editor-in-Chief 